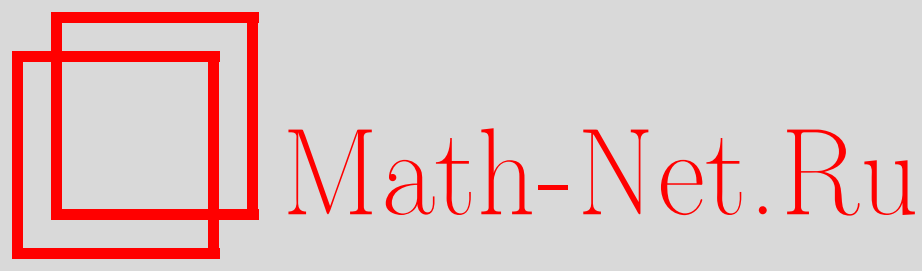

В. А. Андреев, Д. М. Давидович, Л. Д. Давидович, Милена Д. Давидович, Милош Д. Давидович, Преобразования масштаба в фазовом пространстве и растянутые состояния гармонического осциллятора, ТМФ, 2017, том 192, номер 1, 164-184

DOI: https://doi.org/10.4213/tmf9232

Использование Общероссийского математического портала Math-Net.Ru подразумевает, что вы прочитали и согласны с пользовательским соглашением http: //www . mathnet.ru/rus/agreement

Параметры загрузки:

IP : 54.224 .135 .184

26 апреля 2023 г., $14: 27: 16$

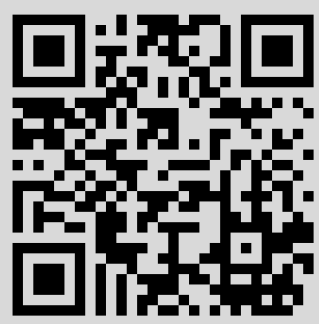




\title{
ФИЗИКА
}

Том 192, № 1

июль, 2017

\section{ПРЕОБРАЗОВАНИЯ МАСШТАБА В ФАЗОВОМ ПРОСТРАНСТВЕ И РАСТЯНУТЫЕ СОСТОЯНИЯ ГАРМОНИЧЕСКОГО ОСЦИЛЛЯТОРА}

\begin{abstract}
Рассматриваются преобразования масштаба $(q, p) \rightarrow(\lambda q, \lambda p)$ в фазовом пространстве. Они индуцируют преобразования функций Хусими $H(q, p)$, заданных на этом пространстве. Рассматриваются функции Хусими состояний, которые являются произвольной суперпозицией $n$-частичных состояний гармонического осциллятора. Развит метод, позволяющий находить так называемые растянутые состояния, в которые преобразуются эти суперпозиции при таком преобразовании масштаба. Изучены их свойства. Вычислены в явном виде матрицы плотности этих растянутых состояний. Установлено, что структуру матриц плотности можно описать, используя отрицательные биномиальные распределения. Для растянутых состояний найдены выражения для их энергии и энтропии и вычислены средние значения оператора числа состояний. Показано, какой вид принимают для растянутых состояний соотношения неопределенностей Гейзенберга и Робертсона-Шредингера.
\end{abstract}

Ключевые слова: фазовое пространство, функция Хусими, преобразование масштаба, гармонический осциллятор, растянутые состояния, соотношения неопределенностей.

DOI: https://doi.org/10.4213/tmf9232

Работа выполнена в рамках научного сотрудничества между РАН и Serbian Academy of Sciences and Arts по теме "Фундаментальные исследования в области квантовой теории информации и квантовых вычислений и их приложения". Bce авторы благодарны Serbian Ministry of Education, Science and Technological Development за поддержку данного проекта. Исследования Л.Д., Милены Д. и Милоша Д. частично поддержаны Serbian Ministry of Education, Science and Technological Development (проекты OI 171031 (Л. Д.) и OI 171028 (М.Д. и М.Д.)).

* Физический институт им. П. Н. Лебедева РАН, Москва, Россия.

E-mail: andrvlad@yandex.ru.

${ }^{\dagger}$ Vinča Institute for Nuclear Sciences, University of Belgrade, Belgrade, Serbia.

E-mail: davidd@vinca.rs, davidovic@vinca.rs

${ }^{\ddagger}$ Institute of Physics, University of Belgrade, Belgrade, Serbia. E-mail: ljubica@ipb.ac.rs

${ }^{\S}$ Faculty of Civil Engineering, University of Belgrade, Belgrade, Serbia.

E-mail: milena@grf.bg.ac.rs 


\section{1. ВВЕДЕНИЕ}

В работе предложен новый метод построения квантовых состояний, возникающих при тех или иных физических процессах. Метод основан на использовании для описания квантовых состояний квазивероятностных распределений. Эти распределения заданы на фазовом пространстве, а физическим процессам сопоставляются некоторые преобразования этого пространства. Преобразования фазового пространства индуцируют преобразования функций, которые заданы на них. Определяя физические состояния, соответствующие преобразованным квазивероятностным распределениям, можно найти результат действия физического процесса на исходное квантовое состояние. Такова общая схема нашего подхода. В настоящей работе в роли квазивероятностных распределений используются функции Хусими, а в качестве физического процесса рассматривается усиление квантовых состояний. Этому процессу можно сопоставить преобразование масштаба в фазовом пространстве.

Известно, что квантовая механика допускает несколько эквивалентных друг другу формулировок. При этом в конкретных задачах одна из них может оказаться более удобной, чем другие, что оправдывает их совместное существование и изучение. Наиболее распространенной является формулировка, в которой квантовому состоянию сопоставляется вектор в гильбертовом пространстве, а наблюдаемым величинам - операторы, действующие в этом пространстве. Другой, также весьма распространенной, формулировкой является формулировка, основанная на использовании так называемых квазивероятностных распределений в фазовом пространстве. Исторически данный подход связан с попытками использовать сходство между статистической природой квантовых явлений и классическими статистическими процессами.

В классической статистической механике физическому состоянию сопоставляется некоторая функция распределения $\rho(q, p)$, заданная на фазовом пространстве системы. Эта функция является плотностью вероятности того, что система находится в состоянии, характеризующемся параметрами $q, p$. Аргументы имеют смысл координаты и импульса, причем $q$ - координата именно той точки пространства, в которой в данный момент времени находится система, и $p$ - именно тот импульс, которым система обладает в данный момент времени. При этом предполагается, что координаты и импульсы можно измерить совместно. Зная функцию распределения системы, можно вычислять ее различные характеристики, например средние значения тех или иных физических величин. С этой целью физической величине также сопоставляется своя функция $F(q, p)$, заданная на фазовом пространстве, и, чтобы найти ее среднее значение в состоянии с функцией распределения $\rho(q, p)$, следует вычислить интеграл

$$
\langle F\rangle=\int F(q, p) \rho(q, p) d q d p .
$$

С самого начала создания квантовой механики предпринимались попытки посмотреть на нее как на статистическую теорию и развить формализм, аналогичный формализму классической статистической теории. А именно, сопоставить квантовому состоянию некоторую функцию $D(q, p)$, которая задана на фазовом пространстве и однозначно его характеризует. Каждому оператору $\hat{A}$, отвечающему наблюдаемой величине, сопоставить свою функцию $A_{D}(q, p)$, также определенную на всем 
фазовом пространстве так, чтобы среднее значение $\langle\hat{A}\rangle$ оператора $\hat{A}$ в состоянии, задаваемом квазивероятностным распределением $D(q, p)$, можно было вычислить с помощью интеграла:

$$
\langle\hat{A}\rangle=\int A_{D}(q, p) D_{\rho}(q, p) d q d p
$$

Функция $D(q, p)$ называется квазивероятностным распределением, которое отвечает данному квантовому состоянию, а функция $A_{D}(q, p)$ - символом оператора $\hat{A}$, построенным по квазивероятностному распределению $D(q, p)$.

В квантовой механике существует целый ряд квазивероятностных распределений, соответственно каждый оператор $\hat{A}$ обладает несколькими символами. Наиболее известными из них являются функции Вигнера $W(q, p)$ [1], Хусими-Кано $Q(q, p)$ [2], [3] и Глаубера-Сударшана $P(q, p)[4],[5]$. Общие свойства квазивероятностных распределений изучались в работах [6]-[9], также разработаны методы нахождения символов операторов, отвечающих этим квазивероятностным распределениям. Вопросы их применения в квантовой оптике рассматривались в книгах [10]-[14].

Помимо различных квазивероятностных распределений, в квантовой механике существует настоящая плотность распределения вероятностей, заданная на фазовом пространстве и полностью определяющая квантовое состояние. Ее называют симплектической томограммой квантового состояния [15]. Свойства томограмм обсуждаются в обзоре [16].

В настоящей статье мы будем работать с функцией Хусими-Кано $Q(q, p)$, которую для краткости будем называть просто функцией Хусими. Ее определение и основные свойства приведены в разделе 2. Основная идея нашего подхода состоит в следующем. Существует ряд квантовых состояний, для которых известен точный аналитический вид их функций Хусими. Мы можем рассмотреть некоторое преобразование фазового пространства $(q, p)$, которое индуцирует преобразование функций Хусими. В результате возникают новые функции, которые зависят от параметров преобразования. Эти новые функции можно попытаться представить в виде суммы уже известных функций Хусими с коэффициентами, зависящими от параметров преобразования фазового пространства. Далее по этой сумме функций Хусими можно найти матрицу плотности преобразованного состояния. В ряде случаев преобразование фазового пространства можно связать с конкретным физическим процессом.

Мы рассматриваем преобразование масштаба в фазовом пространстве вида

$$
(q, p) \rightarrow(\lambda q, \lambda p), \quad|\lambda|^{2} \leqslant 1
$$

В работе [17] было доказано, что если $Q(q, p)$ - функция Хусими квантового состояния и $\lambda<1$, то

$$
Q_{\lambda}(q, p)=\lambda^{2} Q(\lambda q, \lambda p)
$$

также является функцией Хусими некоторого квантового состояния.

В разделе 3 мы показываем, что преобразование функций Хусими (4) естественным образом возникает в задачах квантовой оптики, и это обуславливает наш интерес к данной проблеме. 
Мы изучаем, что происходит с состояниями гармонического осциллятора при таком преобразовании. В разделе 4 рассматривается чистое состояние, являющееся произвольной суперпозицией $n$-частичных состояний. Показано, что в результате преобразования чистое состояние переходит в смешанное, и найдена его матрица плотности. Это смешанное $\lambda$-состояние содержит бесконечный набор чистых состояний, и вероятности, с которыми эти чистые состояния входят в смешанное, образуют отрицательное биномиальное распределение.

В случае, когда изначально мы имеем дело с одним $N$-частичным состоянием, преобразованное $\lambda$-состояние содержит все $M$-частичные состояния с $M \geqslant N$. При этом распределение данных чистых состояний в смешанном становится тем более гладким, чем меньше параметр $\lambda^{2}$. Условно говоря, можно считать, что при $\lambda$-преобразовании (3) из состояния $|N\rangle$ рождаются состояния с большими $M>N$. Мы будем называть такие смешанные состояния растянутыми состояниями. В разделе 5 найдены средние значения оператора числа частиц для растянутых состояний. В разделе 6 вычислена энтропия фон Неймана растянутых состояний. В разделе 7 найден вид соотношений неопределенностей Гейзенберга и Робертсона-Шредингера для растянутых состояний. Показано, что для таких состояний в правой части соотношений неопределенностей появляется множитель $\lambda^{-4}$, т. е. при выполнении $\lambda$-преобразования (3) неопределенность состояний возрастает. Кратко обсуждаются возможные физические следствия этого факта.

Всюду в данной работе мы предполагаем, что $|\lambda|<1$. Однако возникает вопрос, что будет происходить и какие состояния могут возникать при $|\lambda|>1$. В разделе 8 мы формально применяем схему рассуждений, которую использовали для $|\lambda|<1$, в случае $|\lambda|>1$. Показано, что в этом случае функции Хусими $n$-частичных состояний гармонического осциллятора преобразуются в такие величины, которые не являются функциями Хусими никаких квантовых состояний.

\section{2. ФУНКЦИИ ХУСИМИ СОСТОЯНИЙ ГАРМОНИЧЕСКОГО ОСЦИЛЛЯТОРА}

Мы рассматриваем одномерный гармонический осциллятор. Его гамильтониан $\widehat{H}$ задается как

$$
\widehat{H}=-\frac{\hbar^{2}}{2} \frac{d^{2}}{d x^{2}}+\frac{\omega^{2}}{2} x^{2}=\frac{1}{2}\left(\hat{p}^{2}+\omega^{2} \hat{q}^{2}\right)=\hbar \omega\left(\hat{a}^{\dagger} \hat{a}+\frac{1}{2}\right),
$$

где операторы координаты $\hat{q}$ и импульса $\hat{p}$, а также операторы рождения и уничтожения $\hat{a}^{\dagger}, \hat{a}$ имеют вид

$$
\begin{gathered}
\hat{p}=-i \hbar \frac{d}{d x}, \quad \hat{q}=x, \\
\hat{a}^{\dagger}=\frac{1}{\sqrt{2 \hbar \omega}}(\hat{p}+i \omega \hat{q}), \quad \hat{a}=\frac{1}{\sqrt{2 \hbar \omega}}(\hat{p}-i \omega \hat{q}) .
\end{gathered}
$$

Для гамильтониана $H n$-частичные состояния $|n\rangle$ являются его собственными функциями:

$$
\widehat{H}|n\rangle=\hbar \omega\left(n+\frac{1}{2}\right)|n\rangle .
$$


Когерентные состояния гармонического осциллятора имеют вид

$$
|\alpha\rangle=e^{-|\alpha|^{2} / 2} \sum_{n=0}^{\infty} \frac{\alpha^{n}}{\sqrt{n !}}|n\rangle .
$$

Здесь $\alpha-$ произвольное комплексное число.

Пусть мы имеем некоторое квантовое состояние, которое определяется оператором плотности $\hat{\rho}$. Тогда с помощью когерентных состояний (8) можно построить его функцию Хусими:

$$
Q\left(\alpha, \alpha^{*}\right)=\frac{1}{\pi} \int\langle\alpha \mid x\rangle \rho(x, y)\langle y \mid \alpha\rangle d x d y
$$

Если квантовое состояние является чистым и описывается волновой функцией $|\psi\rangle$, то его функция Хусими имеет вид

$$
Q(q, p)=\langle\alpha \mid \psi\rangle\langle\psi \mid \alpha\rangle
$$

Функция Хусими задана на фазовом пространстве с координатами $(q, p)$. В работе [17] рассматривалось преобразование масштаба $(q, p) \rightarrow(\lambda q, \lambda p)$ в этом пространстве. Было показано, что если $Q(q, p)$ - функция Хусими квантового состояния, то $\lambda^{2} Q(\lambda q, \lambda p)$ также есть функция Хусими некоторого квантового состояния при условии, что $|\lambda|^{2} \leqslant 1$.

Ниже мы показываем, что преобразование (3) можно связать с некоторыми физическими процессами, например с прохождением состояния электромагнитного поля через квантовый усилитель [18], [19]. Поэтому представляет интерес задача о нахождении явного вида таких преобразований для конкретных состояний и изучении их свойств. В ряде случаев эту задачу можно решить точно.

В настоящей работе мы рассматриваем гармонический осциллятор и находим матрицы плотности тех состояний, в которые переходят суперпозиции его $n$-частичных состояний при преобразовании масштаба (3). Для этого мы используем специальный метод, основанный на использовании некоторых свойств функций Хусими таких состояний.

\section{3. СВЯЗЬ ПРЕОБРАЗОВАНИЙ МАСШТАБА С ЗАДАЧАМИ КВАНТОВОЙ ОПТИКИ}

Прежде чем переходить к систематическому развитию формализма, укажем на связь данного подхода с задачами квантовой оптики и объясним, как в такой постановке задачи возникает преобразование масштаба (3). Основную идею можно понять на примере простейшего линейного усилителя света, состоящего из частично инвертированных двухуровневых атомов. Резонансный гамильтониан взаимодействия поля с атомами имеет вид

$$
\widehat{H}=\hbar k\left(\begin{array}{cc}
0 & \hat{a} \\
\hat{a}^{\dagger} & 0
\end{array}\right) .
$$

Это гамильтониан взаимодействия в модели Джейнса-Каммингса; он обладает рядом интересных свойств, в частности свойством суперсимметрии [20]. При таком 
гамильтониане уравнение для матрицы плотности $\hat{\rho}$ электромагнитного поля в первом приближении записывается как [13]

$$
\frac{\partial \hat{\rho}}{\partial t}=-k N_{1}\left(\hat{a} \hat{a}^{\dagger} \hat{\rho}-2 \hat{a}^{\dagger} \hat{\rho} \hat{a}+\hat{\rho} \hat{a} \hat{a}^{\dagger}\right)-k N_{2}\left(\hat{a}^{\dagger} \hat{a} \hat{\rho}-2 \hat{a} \hat{\rho} \hat{a}^{\dagger}+\hat{\rho} \hat{a}^{\dagger} \hat{a}\right)
$$

Здесь $\hat{a}^{\dagger}$ и $\hat{a}$ - операторы рождения и уничтожения электромагнитного поля, $N_{1}$ и $N_{2}$ - заселенности верхнего и нижнего уровней двухуровневых атомов, $k$ - коэффициент усиления.

Используя связь (9) между матрицей плотности и функцией Хусими, можно перейти от операторного уравнения (12) к обыкновенному дифференциальному уравнению для функции Хусими. С помощью этого уравнения в работе [18] было получено выражение для функции Хусими для состояния на выходе из квантового усилителя:

$$
Q_{\text {out }}(\alpha, t)=\frac{1}{G^{2}} Q_{\text {in }}\left(\frac{\alpha}{G}\right)=\left\langle\frac{\alpha}{G}\left|\hat{\rho}_{\text {in }}\right| \frac{\alpha}{G}\right\rangle
$$

где

$$
G(t)=e^{2\left(N_{1}-N_{2}\right) k t}
$$

Мы видим, что выражение (13) совпадает с (4) при $\lambda=G^{-1}$. Таким образом, преобразование масштаба (3) в фазовом пространстве оказывается связанным с действием квантового усилителя, и вид этого преобразования определяется структурой гамильтониана (11). Соответственно и действие усилителя на произвольное квантовое состояние можно описать с помощью преобразования масштаба в фазовом пространстве.

Формально в нашей работе мы будем иметь дело только с состояниями гармонического осциллятора, однако, имея в виду, что развиваемый в статье аппарат предполагается использовать для задач квантовой оптики, мы будем иногда называть их $n$-фотонными или $n$-частичными фоковскими состояниями.

\section{4. МАТРИЦЫ ПЛОТНОСТИ РАСТЯНУТЫХ СОСТОЯНИЙ}

Рассмотрим сначала $N$-частичное состояние $|N\rangle$. Его функция Хусими имеет вид

$$
Q_{N}(q, p)=\langle\alpha \mid N\rangle\langle N \mid \alpha\rangle=e^{-|\alpha|^{2}} \frac{|\alpha|^{2 N}}{N !}
$$

После преобразования масштаба (3) получаем

$$
Q_{N}^{\lambda}(q, p)=\lambda^{2} e^{-\lambda^{2}|\alpha|^{2}} \frac{\lambda^{2 N}|\alpha|^{2 N}}{N !} .
$$

Мы хотим представить выражение (16) в виде суммы функций Хусими $Q_{j}(q, p)$ с различными $j$. Для этого запишем его в виде

$$
Q_{N}^{\lambda}(q, p)=e^{-\lambda^{2}|\alpha|^{2}} \frac{\lambda^{2 N+2}|\alpha|^{2 N}}{N !}=e^{-|\alpha|^{2}} e^{\left(1-\lambda^{2}\right)|\alpha|^{2}} \frac{\lambda^{2 N+2}|\alpha|^{2 N}}{N !} .
$$


Теперь разложим в ряд экспоненту $e^{\left(1-\lambda^{2}\right)|\alpha|^{2}}$, получим

$$
\begin{aligned}
Q_{N}^{\lambda}(q, p) & =e^{-|\alpha|^{2}} \sum_{j=0}^{\infty} \frac{\left(1-\lambda^{2}\right)^{j}|\alpha|^{2 j}}{j !} \frac{\lambda^{2 N+2}|\alpha|^{2 N}}{N !}= \\
& =\sum_{j=0}^{\infty} \lambda^{2 N+2} \frac{\left(1-\lambda^{2}\right)^{j}(N+j) !}{j ! N !} e^{-|\alpha|^{2}} \frac{|\alpha|^{2(N+j)}}{(N+j) !}= \\
& =\sum_{j=0}^{\infty} \lambda^{2 N+2} \frac{\left(1-\lambda^{2}\right)^{j}(N+j) !}{j ! N !} Q_{N+j}(q, p) .
\end{aligned}
$$

Переходя теперь от функции Хусими к матрице плотности, получаем, что состоянию (16) соответствует матрица плотности

$$
\hat{\rho}_{N}^{\lambda}=\sum_{j=0}^{\infty} \lambda^{2 N+2} \frac{\left(1-\lambda^{2}\right)^{j}(N+j) !}{j ! N !}|N+j\rangle\langle N+j| .
$$

Эта матрица плотности имеет диагональный вид, при этом первые $N$ диагональных элементов, имеющие номера $0,1, \ldots, N-1$, равны нулю, а дальше на главной диагонали стоят величины

$$
F_{N+j}^{N}=\frac{\left(1-\lambda^{2}\right)^{j}(N+j) !}{j ! N !} \lambda^{2 N+2}, \quad j=0,1, \ldots
$$

Величины (20) образуют отрицательное биномиальное распределение. Элементы этого распределения задаются формулами [21]

$$
f(k, r, p)=\left(\begin{array}{c}
r+k-1 \\
k
\end{array}\right) p^{r} q^{k}=\frac{(r+k-1) !}{(r-1) ! k !} p^{r}(1-p)^{k}, \quad k=0,1,2, \ldots
$$

Они определяются двумя параметрами $r$ и $p, q=1-p$, а число $k$ - номер элемента в распределении. В нашем случае $r=N+1, k=j, p=\lambda^{2}$. Таким образом, мы имеем

$$
F_{N+j}^{N}=f\left(j, N+1, \lambda^{2}\right)
$$

Рассмотрим теперь состояние, которое является суперпозицией двух $k$-частичных состояний $|M\rangle$ и $|N\rangle$. Его волновая функция и матрица плотности имеют вид

$$
\begin{gathered}
\psi_{M, N}=c_{M}|M\rangle+c_{N}|N\rangle, \quad\left|c_{M}\right|^{2}+\left|c_{N}\right|^{2}=1, \\
\hat{\rho}_{M, N}=\left(c_{M}|M\rangle+c_{N}|N\rangle\right)\left(c_{M}^{*}\langle M|+c_{N}^{*}\langle N|\right) .
\end{gathered}
$$


Найдем матрицу плотности растянутого состояния $\hat{\rho}_{M, N}^{\lambda}$. Функция Хусими состояния (23) записывается как

$$
\begin{aligned}
Q_{M, N}(\alpha) & =\left\langle\alpha\left|\rho_{M, N}\right| \alpha\right\rangle= \\
& =e^{-|\alpha|^{2} / 2}\left(c_{M} \frac{\left(\alpha^{*}\right)^{M}}{\sqrt{M !}}+c_{N} \frac{\left(\alpha^{*}\right)^{N}}{\sqrt{N !}}\right) e^{-|\alpha|^{2} / 2}\left(c_{M}^{*} \frac{\alpha^{M}}{\sqrt{M !}}+c_{N}^{*} \frac{\alpha^{N}}{\sqrt{N !}}\right) .
\end{aligned}
$$

После преобразования (3) получаем

$$
\begin{aligned}
& Q_{M, N}^{\lambda}(\lambda q, \lambda p)=\lambda^{2} e^{-\lambda^{2}|\alpha|^{2}}\left(c_{M} \frac{\lambda^{M}\left(\alpha^{*}\right)^{M}}{\sqrt{M !}}+c_{N} \frac{\lambda^{N}\left(\alpha^{*}\right)^{N}}{\sqrt{N !}}\right)\left(c_{M}^{*} \frac{\lambda^{M} \alpha^{M}}{\sqrt{M !}}+c_{N}^{*} \frac{\lambda^{N} \alpha^{N}}{\sqrt{N !}}\right)= \\
& =e^{-|\alpha|^{2}} \lambda^{2} e^{\left(1-\lambda^{2}\right)|\alpha|^{2}}\left(c_{M} \frac{\lambda^{M}\left(\alpha^{*}\right)^{M}}{\sqrt{M !}}+c_{N} \frac{\lambda^{N}\left(\alpha^{*}\right)^{N}}{\sqrt{N !}}\right)\left(c_{M}^{*} \frac{\lambda^{M} \alpha^{M}}{\sqrt{M !}}+c_{N}^{*} \frac{\lambda^{N} \alpha^{N}}{\sqrt{N !}}\right) .
\end{aligned}
$$

Как и в предыдущем случае, разложим в ряд экспоненту $e^{\left(1-\lambda^{2}\right)|\alpha|^{2}}$, тогда функции Хусими (25) примет вид

$$
\begin{aligned}
& Q_{M, N}^{\lambda}(\lambda q, \lambda p)= \\
& =e^{-|\alpha|^{2}} \lambda^{2} \sum_{j=0}^{\infty} \frac{\left(1-\lambda^{2}\right)^{j}|\alpha|^{2 j}}{j !}\left(c_{M} \frac{\lambda^{M}\left(\alpha^{*}\right)^{M}}{\sqrt{M !}}+c_{N} \frac{\lambda^{N}\left(\alpha^{*}\right)^{N}}{\sqrt{N !}}\right)\left(c_{M}^{*} \frac{\lambda^{M} \alpha^{M}}{\sqrt{M !}}+c_{N}^{*} \frac{\lambda^{N} \alpha^{N}}{\sqrt{N !}}\right) .
\end{aligned}
$$

Теперь надо принять во внимание то, что имеют место соотношения

$$
e^{-|\alpha|^{2} / 2} \frac{\alpha^{s+j}}{\sqrt{(s+j) !}}=\langle s+j \mid \alpha\rangle, \quad e^{-|\alpha|^{2} / 2} \frac{\left(\alpha^{*}\right)^{k+j}}{\sqrt{(k+j) !}}=\langle\alpha \mid k+j\rangle .
$$

Используя их, получаем выражение для функции Хусими (25):

$$
\begin{aligned}
& Q_{N, M}^{\lambda}(\lambda q, \lambda p)= \\
& =\sum_{j=0}^{\infty} \frac{\lambda^{2}\left(1-\lambda^{2}\right)^{j}}{j !}\left\langle\alpha \left|\left(\sqrt{\frac{(M+j) !}{M !}} \lambda^{M} c_{M}|M+j\rangle+\sqrt{\frac{(N+j) !}{N !}} \lambda^{N} c_{N}|N+j\rangle\right) \times\right.\right. \\
& \quad \times\left(\sqrt{\frac{(M+j) !}{M !}} \lambda^{M} c_{M}^{*}\left\langle M+j\left|+\sqrt{\frac{(N+j) !}{N !}} \lambda^{N} c_{N}^{*}\langle N+j|\right) \mid \alpha\right\rangle .\right.
\end{aligned}
$$

Отсюда следует, что матрица плотности растянутого состояния $\hat{\rho}_{M, N}^{\lambda}$ такова:

$$
\begin{aligned}
\hat{\rho}_{N, M}^{\lambda}= & \sum_{j=0}^{\infty} \frac{\lambda^{2}\left(1-\lambda^{2}\right)^{j}}{j !}\left(\sqrt{\frac{(M+j) !}{M !}} \lambda^{M} c_{M}|M+j\rangle+\sqrt{\frac{(N+j) !}{N !}} \lambda^{N} c_{N}|N+j\rangle\right) \times \\
& \times\left(\sqrt{\frac{(M+j) !}{M !}} \lambda^{M} c_{M}^{*}\langle M+j|+\sqrt{\frac{(N+j) !}{N !}} \lambda^{N} c_{N}^{*}\langle N+j|\right) .
\end{aligned}
$$

Рассмотрим структуру этой матрицы. 
Матрица плотности (28) имеет три ненулевые диагонали. Пусть $M=N+k$, тогда на главной диагонали первые $N$ элементов, имеющие номера $0,1, \ldots, N-1$, равны нулю. Следующие $k$ диагональных элементов с номерами $N, \ldots, N+k-1$ совпадают с первыми $k$ ненулевыми диагональными элементами матрицы плотности (19),

$$
D_{N+j, N+j}=\frac{\left(1-\lambda^{2}\right)^{j}(N+j) !}{j ! N !} \lambda^{2+2 N}\left|c_{N}\right|^{2}, \quad j=0,1, \ldots, k-1 .
$$

Остальные элементы главной диагонали матрицы плотности (28) имеют вид суммы диагональных элементов матриц плотности $\rho_{N}^{\lambda}$ и $\rho_{M}^{\lambda}$, заданных формулой (19):

$$
\begin{aligned}
D_{N+k+j, N+k+j}= & \frac{\left(1-\lambda^{2}\right)^{k+j}(N+k+j) !}{(k+j) ! N !} \lambda^{2+2 N}\left|c_{N}\right|^{2}+ \\
& +\frac{\left(1-\lambda^{2}\right)^{j}(M+j) !}{j ! M !} \lambda^{2+2 M}\left|c_{M}\right|^{2}, \quad j=0,1, \ldots
\end{aligned}
$$

Таким образом, главная диагональ матрицы плотности $\hat{\rho}_{N, M}^{\lambda}(28)$ имеет вид суммы главных диагоналей матриц плотности $\hat{\rho}_{N}^{\lambda}$ и $\hat{\rho}_{M}^{\lambda}$, умноженных на коэффициенты $\left|c_{N}\right|^{2}$ и $\left|c_{M}\right|^{2}$.

Помимо главной диагонали, матрица плотности (28) имеет ненулевые элементы еще на двух диагоналях, расположенных сверху и снизу от главной диагонали на расстоянии $k$ шагов. Эти ненулевые элементы расположены в ячейках с координатами $(N+i, N+k+i),(N+k+i, N+i), i=0,1, \ldots$. Элементы матрицы плотности с координатами $(N+j, M+j),(M+j, N+j)$ имеют вид

$$
\begin{aligned}
& D_{N+j, M+j}=\frac{\left(1-\lambda^{2}\right)^{j}}{j !} \lambda^{N+M+2} \sqrt{\frac{(N+j) !(M+j) !}{N ! M !}} c_{N} c_{M}^{*}, \\
& D_{M+j, N+j}=\frac{\left(1-\lambda^{2}\right)^{j}}{j !} \lambda^{N+M+2} \sqrt{\frac{(N+j) !(M+j) !}{N ! M !}} c_{N}^{*} c_{M},
\end{aligned}
$$

Теперь мы можем рассмотреть общий случай произвольного состояния гармонического осциллятора. Схема рассуждений остается той же, что и в случае состояния (19), которое является суперпозицией двух фоковских состояний.

Рассмотрим чистое состояние, являющееся произвольной суперпозицией $n$-частичных состояний гармонического осциллятора:

$$
|\psi\rangle_{\Sigma}=\sum_{k=0}^{\infty} c_{k}|k\rangle, \quad \sum_{k=0}^{\infty}\left|c_{k}\right|^{2}=1
$$

Его функция Хусими имеет вид

$$
Q_{\Sigma}(q, p)=\langle\alpha \mid \psi\rangle\langle\psi \mid \alpha\rangle=e^{-|\alpha|^{2} / 2} \sum_{k=0}^{\infty} \frac{\left(\alpha^{*}\right)^{k}}{\sqrt{k !}} c_{k} e^{-|\alpha|^{2} / 2} \sum_{s=0}^{\infty} \frac{\alpha^{s}}{\sqrt{s !}} c_{s}^{*} .
$$


После $\lambda$-преобразования масштаба функция Хусими (33) приобретает вид

$$
\begin{aligned}
Q_{\Sigma}^{\lambda}(q, p)=\lambda^{2} Q(\lambda q, \lambda p) & =\lambda^{2} e^{-\lambda^{2}|\alpha|^{2}} \sum_{k=0}^{\infty} \frac{\lambda^{k}\left(\alpha^{*}\right)^{k}}{\sqrt{k !}} c_{k} \sum_{s=0}^{\infty} \frac{\lambda^{s} \alpha^{s}}{\sqrt{s !}} c_{s}^{*}= \\
& =e^{-|\alpha|^{2}} \lambda^{2} e^{\left(1-\lambda^{2}\right)|\alpha|^{2}} \sum_{k=0}^{\infty} \frac{\lambda^{k}\left(\alpha^{*}\right)^{k}}{\sqrt{k !}} c_{k} \sum_{s=0}^{\infty} \frac{\lambda^{s} \alpha^{s}}{\sqrt{s !}} c_{s}^{*} .
\end{aligned}
$$

Разложим в ряд экспоненту $e^{\left(1-\lambda^{2}\right)|\alpha|^{2}}$, тогда выражение $(34)$ примет вид

$$
\lambda^{2} Q_{\Sigma}(\lambda q, \lambda p)=e^{-|\alpha|^{2}} \lambda^{2} \sum_{j=0}^{\infty} \frac{\left(1-\lambda^{2}\right)^{j}|\alpha|^{2 j}}{j !} \sum_{k=0}^{\infty} \frac{\lambda^{k}\left(\alpha^{*}\right)^{k}}{\sqrt{k !}} c_{k} \sum_{s=0}^{\infty} \frac{\lambda^{s} \alpha^{s}}{\sqrt{s !}} c_{s}^{*} .
$$

Его можно записать как

$$
\lambda^{2} Q_{\Sigma}(\lambda q, \lambda p)=e^{-|\alpha|^{2}} \sum_{j=0}^{\infty} \lambda^{2} \frac{\left(1-\lambda^{2}\right)^{j}}{j !} \sum_{k=0}^{\infty} \frac{\lambda^{k}\left(\alpha^{*}\right)^{k+j}}{\sqrt{k !}} c_{k} \sum_{s=0}^{\infty} \frac{\lambda^{s} \alpha^{s+j}}{\sqrt{s !}} c_{s}^{*} .
$$

Теперь, как и прежде, следует учесть соотношения (26), используя которые, мы запишем равенство (36) в виде

$$
\begin{aligned}
& \lambda^{2} Q_{\Sigma}(\lambda q, \lambda p)= \\
& \quad=\sum_{j=0}^{\infty} \lambda^{2} \frac{\left(1-\lambda^{2}\right)^{j}}{j !} \sum_{k=0}^{\infty} \sqrt{\frac{(k+j) !}{k !}} \lambda^{k} c_{k}\langle\alpha \mid k+j\rangle \sum_{s=0}^{\infty} \sqrt{\frac{(s+j) !}{s !}} \lambda^{s} c_{s}^{*}\langle s+j \mid \alpha\rangle .
\end{aligned}
$$

Мы видим, что выражение (37) есть функция Хусими состояния с матрицей плотности

$$
\hat{\rho}_{\Sigma}^{\lambda}=\sum_{j=0}^{\infty} \frac{\lambda^{2}\left(1-\lambda^{2}\right)^{j}}{j !}\left(\sum_{k=0}^{\infty} \sqrt{\frac{(k+j) !}{k !}} \lambda^{k} c_{k}|k+j\rangle\right)\left(\sum_{s=0}^{\infty} \sqrt{\frac{(s+j) !}{s !}} \lambda^{s} c_{s}^{*}\langle s+j|\right) .
$$

Мы получили, что после $\lambda$-преобразования масштаба (3) чистое состояние (32) переходит в смешанное состояние, которое описывается матрицей плотности (38).

Рассмотрим теперь структуру матрицы плотности (38). Для этого в качестве образца следует использовать матрицу плотности (28), соответствующую растянутому состоянию, которое получается из состояния, являющегося суперпозицией двух фоковских состояний. Для такого состояния главная диагональ матрицы плотности имеет вид суммы главных диагоналей матриц плотности, отвечающих одиночным фоковским состояниям, элементы каждой из которых умножены на квадрат модуля того коэффициента, с которым это одиночное состояние входит в суперпозицию.

Если же состояние (32) является суперпозицией не двух, а большего числа $n$-частичных состояний, то главная диагональ матрицы плотности (38) соответствующего растянутого состояния устроена аналогичным образом. А именно, она имеет вид суммы главных диагоналей матриц плотности, отвечающих одиночным $n$-частичным состояниям, элементы каждой из которых умножены на квадрат модуля $\left|c_{k}\right|^{2}$ того коэффициента, с которым это одиночное состояние $|k\rangle$ входит в суперпозицию (32). 
Введем обозначения

$$
\begin{aligned}
& d_{N, j}=\lambda^{N+1} \sqrt{\frac{\left(1-\lambda^{2}\right)^{j}(N+j) !}{N ! j !}} c_{N}, \\
& d_{N, j}^{*}=\lambda^{N+1} \sqrt{\frac{\left(1-\lambda^{2}\right)^{j}(N+j) !}{N ! j !}} c_{N}^{*},
\end{aligned}
$$

В этих обозначениях элементы, лежащие на главной диагонали матрицы плотности (28), принимают вид

$$
D_{n, n}=\sum_{i=0}^{n}\left|d_{i, n-i}\right|^{2}, \quad i=0,1, \ldots, n, \quad n=0,1, \ldots
$$

Ненулевые элементы матрицы плотности (38) лежат, помимо главной диагонали, также на других диагоналях, параллельных главной. Их структура аналогична структуре боковых диагоналей матрицы плотности (28). Опишем элементы, которые стоят на этих диагоналях. Для этого мы будем пользоваться обозначениями (31). Фактически эти элементы являются суммами величин типа (31).

Сначала рассмотрим диагональ, ближайшую к главной диагонали матрицы (28) и расположенную выше нее. Ее элементы имеют координаты $(n, n+1), n=0,1,2, \ldots$, и задаются формулой

$$
D_{n, n+1}=\sum_{j=0}^{n} d_{j, n-j} d_{j+1, n-j}^{*}
$$

Пределы суммирования в этой формуле определяются тем, какие $k$-частичные состояния входят в состояние суперпозиции (32). Если состояние (32) содержит конечное число $k$-частичных состояний и самым большим из них является состояние $|K\rangle$, то формула (41) принимает вид

$$
D_{n, n+1}= \begin{cases}\sum_{j=0}^{n} d_{j, n-j} d_{j+1, n-j}^{*}, & n<K, \\ \sum_{j=0}^{K-1} d_{j, n-j} d_{j+1, n-j}^{*}, & n \geqslant K .\end{cases}
$$

Значения элементов, которые расположены на других диагоналях, проходящих выше главной диагонали, задаются как

$$
D_{n, n+k}= \begin{cases}\sum_{j=0}^{n} d_{j, n-j} d_{j+k, n-j}^{*}, & n \leqslant K-k, \\ \sum_{j=0}^{K-k} d_{j, n-j} d_{j+k, n-j}^{*}, & n \geqslant K-k .\end{cases}
$$

Если какие-то слагаемые в состоянии (32) отсутствуют, т. е. некоторые коэффициенты $c_{k}=0$, то и отвечающие им величины $d_{k, j}=0, j=0,1, \ldots$, и соответствующие 
слагаемые в сумме (39) отсутствуют. Значения элементов матрицы плотности, расположенных ниже главной диагонали, определяются из ее свойства эрмитовости:

$$
D_{n+k, n}=D_{n, n+k}^{*}
$$

Так устроена матрица плотности (38) растянутого состояния, соответствующего состоянию (32). Далее мы изучим некоторые ее свойства, но сначала отметим следующий факт. Элементы, лежащие на главной диагонали матрицы (38), являются линейными комбинациями членов отрицательных биномиальных распределений (21). В нашем случае эти распределения отличаются друг от друга параметром $N$, а параметр $\lambda$ у всех один и тот же. На диагоналях, расположенных выше и ниже главной диагонали, стоят элементы, которые являются линейными комбинациями членов распределений, имеющих вид

$$
F(N, M ; \lambda)_{j}=\frac{\left(1-\lambda^{2}\right)^{j}}{j !} \lambda^{N+M+2} \sqrt{\frac{(N+j) !(M+j) !}{N ! M !}}, \quad j=0,1, \ldots
$$

Распределения (45) характеризуются тремя параметрами $N, M, \lambda$, где $N$ и $M-$ целые числа, а $\lambda^{2} \leqslant 1$. При $N=M$ они переходят в обычные отрицательные биномиальные распределения. Мы будем называть распределения (45) двойными отрицательными биномиальными распределениями. Их свойства будут изучены в отдельной работе.

\section{5. СРЕДНИЕ ЗНАЧЕНИЯ ОПЕРАТОРА ЧИСЛА СОСТОЯНИЙ}

Изучим теперь некоторые свойства растянутых состояний. Прежде всего найдем среднее значение числа частиц растянутого состояния (38). Сначала сделаем это для состояния (21).

Среднее число частиц в состоянии, которое задается матрицей плотности $\rho$, определяется выражением

$$
\langle\hat{n}\rangle=\operatorname{Tr}(\hat{N} \hat{\rho})
$$

где $\hat{N}=\hat{a}^{+} \hat{a}-$ оператор числа частиц.

Для состояния (21) имеем

$$
\left\langle\hat{n}_{N}\right\rangle=\sum_{j=0}^{\infty} \frac{\lambda^{2}\left(1-\lambda^{2}\right)^{j}}{j !}\left(\frac{(N+j) !}{N !} \lambda^{2 N}(N+j)\right) .
$$

Известно, что справедливо соотношение

$$
S_{N}^{0}(x)=\sum_{j=0}^{\infty} \frac{(N+j) !}{j !} x^{j}=\frac{N !}{(1-x)^{N+1}}, \quad|x|<1 .
$$

Подставляя в него $x=1-\lambda^{2}$, получаем

$$
\sum_{j=0}^{\infty} \lambda^{2 N+2} \frac{(N+j) !}{N ! j !}\left(1-\lambda^{2}\right)^{j}=1 \text {. }
$$


Вычислим теперь величину

$$
S_{N}^{1}(x)=\sum_{j=0}^{\infty} j \frac{(N+j) !}{j !} x^{j}=x \frac{d}{d x} S_{N}^{0}(x)=\frac{x(N+1) !}{(1-x)^{N+2}} .
$$

Используя соотношения (49), (50), получаем выражение для среднего значения числа частиц в растянутом состоянии (21):

$$
\left\langle\hat{n}_{N}\right\rangle=N+\left(S_{N}^{0}\right)^{-1} S_{N}^{1}=N+\frac{(N+1)\left(1-\lambda^{2}\right)}{\lambda^{2}}=\frac{N+1}{\lambda^{2}}-1 .
$$

Найдем выражение для дисперсии числа частиц в состоянии (21). Эта величина задается как

$$
\sigma_{n}=\left\langle\hat{n}^{2}\right\rangle-\langle\hat{n}\rangle^{2}
$$

Чтобы вычислить ее значение, надо знать среднее $\left\langle\hat{n}_{N}^{2}\right\rangle$. Найдем его:

$$
\begin{aligned}
\left\langle\hat{n}_{N}^{2}\right\rangle & =\sum_{j=0}^{\infty} \lambda^{2 N+2} \frac{\left(1-\lambda^{2}\right)^{j}}{j !} \frac{(N+j) !}{N !}(N+j)^{2}= \\
& =N^{2}+2 N\left\langle n_{N}\right\rangle+\sum_{j=0}^{\infty} \lambda^{2 N+2} \frac{\left(1-\lambda^{2}\right)^{j}}{j !} \frac{(N+j) !}{N !} j^{2} .
\end{aligned}
$$

Далее мы имеем

$$
\begin{aligned}
S_{N}^{2}(x) & =\sum_{i=0}^{\infty} i^{2} \frac{(N+i) !}{i !} x^{i}= \\
& =x \frac{d}{d x} S_{1}=\left(x \frac{d}{d x}\right)^{2} S_{0}=\frac{x(N+1)}{1-x}+\frac{x^{2}(N+1)(N+2)}{(1-x)^{2}} .
\end{aligned}
$$

Отсюда, пользуясь соотношениями (51), получаем выражение для дисперсии числа частиц в состоянии (21):

$$
\sigma_{n}=\left\langle\hat{n}_{N}^{2}\right\rangle-\left\langle\hat{n}_{N}\right\rangle^{2}=\frac{x(N+1)}{(1-x)^{2}}=\frac{(N+1)\left(1-\lambda^{2}\right)}{\lambda^{4}} .
$$

Мы нашли значения $\left\langle\hat{n}_{N}\right\rangle$ и $\left\langle\hat{n}_{N}^{2}\right\rangle$, получим теперь $\left\langle\hat{n}_{N}^{l}\right\rangle$ для состояния (21). В этом случае мы имеем

$$
\begin{aligned}
\left\langle\hat{n}_{N}^{l}\right\rangle & =\sum_{j=0}^{\infty} \lambda^{2 N+2} \frac{\left(1-\lambda^{2}\right)^{j}}{j !} \frac{(N+j) !}{N !}(N+j)^{l}= \\
& =\sum_{j=0}^{\infty} F_{N+j}^{N} \sum_{k=0}^{l}\left(\begin{array}{l}
l \\
k
\end{array}\right) j^{k} N^{l-k}=\sum_{k=0}^{l} G_{N}^{k}(\lambda)\left(\begin{array}{l}
l \\
k
\end{array}\right) N^{l-k} .
\end{aligned}
$$

Здесь $\left(\begin{array}{l}l \\ k\end{array}\right)=\frac{l !}{(l-k) ! k !}-$ обычные биномиальные коэффициенты, а величины $G_{N}^{k}(\lambda)$ имеют вид

$$
G_{N}^{k}(\lambda)=\sum_{j=0}^{\infty} \lambda^{2 N+2} \frac{\left(1-\lambda^{2}\right)^{j}}{j !} \frac{(N+j) !}{N !} j^{k}
$$


Вычислим эти суммы. Как мы уже показали, имеет место равенство (48). Используя его, получаем

$$
\begin{aligned}
S_{N}^{1}(x) & =\sum_{i=0}^{\infty} i \frac{(N+i) !}{i !} x^{i}=x \frac{d}{d x} S_{0}, \\
S_{N}^{2}(x) & =\sum_{i=0}^{\infty} i^{2} \frac{(N+i) !}{i !} x^{i}=x \frac{d}{d x} S_{1}=\left(x \frac{d}{d x}\right)^{2} S_{0}, \\
& \ldots \ldots \ldots \ldots \ldots \ldots \ldots \ldots \ldots \ldots \\
S_{N}^{k}(x) & =\sum_{i=0}^{\infty} i^{k} \frac{(N+i) !}{i !} x^{i}=\left(x \frac{d}{d x}\right)^{k} S_{0},
\end{aligned}
$$

Используя эти соотношения, получаем выражение для величин $G_{N}^{k}(\lambda)$ :

$$
G_{N}^{k}(\lambda)=\left(S_{N}^{0}\left(1-\lambda^{2}\right)\right)^{-1} S_{N}^{k}\left(1-\lambda^{2}\right) .
$$

Таким образом, для $\left\langle\hat{n}_{N}^{l}\right\rangle$ мы имеем следующую формулу:

$$
\left\langle\hat{n}^{l}\right\rangle=\sum_{k=0}^{l}\left(S_{N}^{0}\left(1-\lambda^{2}\right)\right)^{-1} S_{N}^{k}\left(1-\lambda^{2}\right)\left(\begin{array}{l}
l \\
k
\end{array}\right) N^{l-k} .
$$

Отметим тот факт, что при построении величины $\left\langle\hat{n}_{N}^{l}\right\rangle$ использовались как положительные, так и отрицательные биномиальные коэффициенты.

Вычислим теперь значения величин (51), (60) для растянутых состояний с матрицей плотности (38). Сначала сделаем это для состояния (28). В этом случае среднее число частиц $\left\langle\hat{n}_{N, V}\right\rangle$ определяется выражением

$$
\begin{aligned}
\left\langle\hat{n}_{N, V}\right\rangle & =\sum_{j=0}^{\infty} \frac{\lambda^{2}\left(1-\lambda^{2}\right)^{j}}{j !}\left(\frac{(N+j) !}{N !} \lambda^{2 N}\left|c_{N}\right|^{2}(N+j)+\frac{(M+j) !}{M !} \lambda^{2 M}\left|c_{M}\right|^{2}(M+j)\right)= \\
& =\frac{1}{\lambda^{2}}\left(\left|c_{N}\right|^{2} N+\left|c_{M}\right|^{2} M\right)+\frac{1}{\lambda^{2}}-1, \quad\left|c_{N}\right|^{2}+\left|c_{M}\right|^{2}=1
\end{aligned}
$$

Среднее значение произвольной степени оператора числа частиц $\left\langle\left(\hat{n}_{N, V}\right)^{l}\right\rangle$ для состояния (28) имеет вид

$$
\begin{aligned}
& \left\langle\left(\hat{n}_{N, V}\right)^{l}\right\rangle= \\
& \quad=\sum_{j=0}^{\infty} \frac{\lambda^{2}\left(1-\lambda^{2}\right)^{j}}{j !}\left(\frac{(N+j) !}{N !} \lambda^{2 N}\left|c_{N}\right|^{2}(N+j)^{l}+\frac{(M+j) !}{M !} \lambda^{2 M}\left|c_{M}\right|^{2}(M+j)^{l}\right)= \\
& \quad=\frac{1}{\lambda^{2}}\left(\left|c_{N}\right|^{2}\left\langle\hat{n}_{N}^{l}\right\rangle+\left|c_{M}\right|^{2}\left\langle\hat{n}_{M}^{l}\right\rangle\right)+\frac{1}{\lambda^{2}}-1, \quad\left|c_{N}\right|^{2}+\left|c_{M}\right|^{2}=1
\end{aligned}
$$

В случае состояния общего вида $(38)$ величи́ны $\left\langle\hat{n}_{\Sigma}\right\rangle$ и $\left\langle\left(\hat{n}_{\Sigma}\right)^{l}\right\rangle$ записываются как

$$
\begin{gathered}
\left\langle\hat{n}_{\Sigma}\right\rangle=\frac{1}{\lambda^{2}} \sum_{k=0}^{\infty}\left|c_{k}\right|^{2} k+\frac{1}{\lambda^{2}}-1, \\
\left\langle\left(\hat{n}_{\Sigma}\right)^{l}\right\rangle=\frac{1}{\lambda^{2}} \sum_{k=0}^{\infty}\left|c_{k}\right|^{2}\left\langle\hat{n}_{k}^{l}\right\rangle+\frac{1}{\lambda^{2}}-1,
\end{gathered}
$$


Мы видим, что формулы (63) для средних значений степеней оператора числа частиц для состояния (38) представляют собой суммы аналогичных выражений для состояния (21). Это объясняется тем, что в формулы (63) входят только диагональные элементы матрицы плотности (38), а эти элементы равны сумме диагональных элементов матриц плотности (21) для растянутых состояний $|k\rangle$, умноженных на квадраты модулей коэффициентов $c_{k}$, с которыми они входят в состояние $(32)$.

\section{6. ЭНТРОПИЯ РАСТЯНУТЫХ СОСТОЯНИЙ}

Рассмотрим теперь энтропию фон Неймана $H$ растянутых состояний. Пусть мы имеем состояние, которое определяется матрицей плотности $\hat{\rho}$, тогда величина $H$ определяется по формуле

$$
H=-\sum_{k} \lambda_{k} \log _{2} \lambda_{k}
$$

Здесь $\lambda_{k}$ - собственные значения матрицы плотности $\rho$. При этом предполагается, что $0 \cdot \log _{2} 0=0$. Из этого определения следует, что энтропия чистого состояния равна нулю, поскольку из свойства идемпотентности матрицы плотности чистого состояния $\hat{\rho}^{2}=\hat{\rho}$ вытекает, что одно ее собственное значение равно единице, а остальные равны нулю.

Если же мы имеем смешанное состояние, то его энтропия определяется распределением величин $\lambda_{k}$. Значение $\lambda_{k}$ определяет вероятность обнаружить систему в том состоянии, которому отвечает данное собственное значение матрицы плотности. Легко понять, что величина энтропии (64) тем больше, чем более равномерно распределены вероятности $\lambda_{k}$, и достигает максимального значения, когда они равны друг другу. В том случае, когда сумма (64) содержит $N$ слагаемых, максимальное значение энтропии фон Неймана $H$ достигается при $\lambda_{k}=1 / N$ и равно $H=\log _{2} N$.

Рассмотрим теперь растянутое состояние, отвечающее $N$-частичному состоянию гармонического осциллятора. Его матрица плотности имеет вид

$$
\hat{\rho}_{N}=\frac{\lambda^{2 N+2}}{N !} \sum_{k=0}^{\infty} \frac{(N+k) !}{k !}\left(1-\lambda^{2}\right)^{k}|N+k\rangle\langle N+k|, \quad \lambda^{2}<1 .
$$

Поскольку эта матрица диагональна, ее элементы

$$
c_{k}^{N}=\frac{\lambda^{2 N+2}(N+k) !}{N ! k !}\left(1-\lambda^{2}\right)^{k}
$$

являются ее собственными значениями, и их можно непосредственно подставлять в выражение (64).

Таким образом, чтобы найти энтропию фон Неймана состояния (65), надо вычислить величину

$$
\begin{aligned}
H_{N} & =-\sum_{k=0}^{\infty} c_{k}^{N} \log _{2} c_{k}^{N}= \\
& =-\sum_{k=0}^{\infty} \frac{\lambda^{2 N+2}(N+k) !}{N ! k !}\left(1-\lambda^{2}\right)^{k} \log _{2}\left(\frac{\lambda^{2 N+2}(N+k) !}{N ! k !}\left(1-\lambda^{2}\right)^{k}\right) .
\end{aligned}
$$


Если $N=0$, то мы имеем

$$
H_{0}=-\lambda^{2} \sum_{k=0}^{\infty}\left(1-\lambda^{2}\right)^{k} \log _{2}\left(\lambda^{2}\left(1-\lambda^{2}\right)^{k}\right)=-\log _{2} \lambda^{2}-\log _{2}\left(1-\lambda^{2}\right) \frac{1-\lambda^{2}}{\lambda^{2}}
$$

Если $\lambda \rightarrow 1$, то $H_{0} \rightarrow 0$, и это отвечает тому, что энтропия чистого $N$-частичного состояния равна нулю. Если $\lambda \rightarrow 0$, то $H_{0} \rightarrow \infty$. Это означает, что неопределенность в вероятности наблюдения произвольного $n$-частичного состояния возрастает.

Если мы имеем растянутое состояние, которое отвечает суперпозиции (32) $n$-частичных состояний гармонического осциллятора, то его матрица плотности (38) не является диагональной, и, чтобы вычислить энтропию такого состояния, надо находить ее собственные значения.

\section{7. СООТНОШЕНИЯ НЕОПРЕДЕЛЕННОСТЕЙ ДЛЯ РАСТЯНУТЫХ СОСТОЯНИЙ}

В предыдущем разделе мы вычислили средние значения оператора числа частиц и его степеней для растянутого состояния, которое получается из произвольной суперпозиции $k$-частичных фоковских состояний гармонического осциллятора. В этом разделе мы рассмотрим некоторые другие операторы и покажем, какой вид принимают соотношения неопределенностей Гейзенберга и Робертсона-Шредингера для растянутых состояний. В работе [22] был предложен новый способ построения символов Хусими. Он оказывается особенно эффективным, когда оператор имеет вид полинома по операторам координаты $\hat{q}$ и импульса $\hat{p}$. Именно с такими операторами мы будем иметь дело в данном разделе.

Рассмотрим сначала гамильтониан гармонического осциллятора

$$
\widehat{H}=\frac{\hbar \omega}{2}\left(\hat{q}^{2}+\hat{p}^{2}\right)
$$

Его символ Хусими $K_{\mathrm{H}}(q, p)$ имеет вид

$$
K_{\mathrm{H}}(q, p)=q^{2}+p^{2}-1
$$

Среднее значение энергии $\bar{E}$ состояния, которое характеризуется функцией Хусими $Q(q, p)$, таково:

$$
\bar{E}=\int \frac{\hbar \omega}{2}\left(q^{2}+p^{2}-1\right) Q(q, p) d q d p=\int \frac{\hbar \omega}{2}\left(q^{2}+p^{2}\right) Q(q, p) d q d p-\frac{\hbar \omega}{2} .
$$

Найдем теперь среднее значение энергии растянутого состояния, которое соответствует состоянию с функцией Хусими $Q(q, p)$. Функция Хусими растянутого состояния записывается как $Q_{\lambda}(q, p)=\lambda^{2} Q(\lambda q, \lambda p)$, и среднее значение энергии $\bar{E}_{\lambda}$ состояния с такой функцией Хусими определяется по формуле

$$
\begin{aligned}
\bar{E}_{\lambda} & =\int K_{\mathrm{H}}(q, p) Q_{\lambda}(q, p) d q d p= \\
& =\int \frac{1}{\lambda^{2}} \frac{\hbar \omega}{2}\left((\lambda q)^{2}+(\lambda p)^{2}-1\right) Q(\lambda q, \lambda p) d(\lambda q) d(\lambda p)=\frac{1}{\lambda^{2}} \bar{E}+\frac{1-\lambda^{2}}{\lambda^{2}} \frac{\hbar \omega}{2} .
\end{aligned}
$$


Это выражение справедливо для всех растянутых состояний гармонического осциллятора. Поскольку $|\lambda|^{2}<1$, мы можем заметить, что при преобразовании масштаба $(q, p) \rightarrow(\lambda q, \lambda p)$ энергия растянутых состояний возрастает. Для фоковских состояний среднее значение энергии соответствующих растянутых состояний можно найти в явном виде. Так, в случае суперпозиции (32) мы имеем

$$
\bar{E}_{\Sigma \lambda}=\frac{1}{\lambda^{2}} \frac{\hbar \omega}{2} \sum_{k=0}^{\infty}\left|c_{k}\right|^{2} k+\frac{1-\lambda^{2}}{\lambda^{2}} \frac{\hbar \omega}{2} .
$$

Используя выражение для средних значений операторов через функции Хусими состояний, можно установить, какую форму приобретают соотношения неопределенностей для растянутых состояний. Мы будем рассматривать соотношения неопределенностей Гейзенберга и Робертсона-Шредингера. В общем виде они записываются каK

$$
\sigma_{q q} \sigma_{p p} \geqslant \frac{1}{4} \hbar^{2}, \quad \sigma_{q q} \sigma_{p p}-\sigma_{q p}^{2} \geqslant \frac{1}{4} \hbar^{2}
$$

и выполняются для любых квантовых состояний. Установим теперь, что происходит с этими соотношениями, когда мы переходим к растянутым состояниям. Для этого нужно вычислить величины

$$
\sigma_{q q}=\left\langle\hat{q}^{2}\right\rangle-\langle\hat{q}\rangle^{2}, \quad \sigma_{p p}=\left\langle\hat{p}^{2}\right\rangle-\langle\hat{p}\rangle^{2}, \quad \sigma_{q p}=\frac{1}{2}\langle\hat{p} \hat{q}+\hat{q} \hat{p}\rangle-\langle\hat{q}\rangle\langle\hat{p}\rangle .
$$

Используя функцию Хусими, дисперсии $\sigma_{q q}, \sigma_{p p}$ и величину $\sigma_{q p}$ можно записать в виде

$$
\begin{gathered}
\sigma_{q q}=\int\left(q^{2}-\frac{1}{2}\right) Q(q, p) d q d p-\left(\int q Q(q, p) d q d p\right)^{2}, \\
\sigma_{p p}=\int\left(p^{2}-\frac{1}{2}\right) Q(q, p) d q d p-\left(\int p Q(q, p) d q d p\right)^{2}, \\
\sigma_{q p}=\int q p Q(q, p) d q d p-\int q Q(q, p) d q d p \int q Q(q, p) d q d p .
\end{gathered}
$$

Для растянутых состояний данные формулы принимают вид

$$
\begin{gathered}
\sigma_{q q \lambda}=\int\left(q^{2}-\frac{1}{2}\right) \lambda^{2} Q(\lambda q, \lambda p) d q d p-\left(\int q \lambda^{2} Q(\lambda q, \lambda p) d q d p\right)^{2}, \\
\sigma_{p p \lambda}=\int\left(p^{2}-\frac{1}{2}\right) \lambda^{2} Q(\lambda q, \lambda p) d q d p-\left(\int p \lambda^{2} Q(\lambda q, \lambda p) d q d p\right)^{2}, \\
\sigma_{q p \lambda}=\int q p \lambda^{2} Q(\lambda q, \lambda p) d q d p-\int q \lambda^{2} Q(\lambda q, \lambda p) d q d p \int q \lambda^{2} Q(\lambda q, \lambda p) d q d p .
\end{gathered}
$$

Эти выражения дают значения величин $\sigma_{q q}, \sigma_{p p}, \sigma_{q p}$ для растянутых состояний:

$$
\sigma_{q q \lambda}=\frac{1}{\lambda^{2}} \sigma_{q q}+\frac{1-\lambda^{2}}{\lambda^{2}}, \quad \sigma_{p p \lambda}=\frac{1}{\lambda^{2}} \sigma_{p p}+\frac{1-\lambda^{2}}{\lambda^{2}}, \quad \sigma_{q p \lambda}=\frac{1}{\lambda^{2}} \sigma_{q p} .
$$


Используя полученные выражения, находим, как модифицируются соотношения неопределенностей Гейзенберга и Робертсона-Шредингера при переходе к растянутым состояниям:

$$
\begin{array}{r}
\sigma_{q q \lambda} \sigma_{p p \lambda}=\frac{1}{\lambda^{4}}\left(\sigma_{q q} \sigma_{p p}+\frac{1}{2}\left(1-\lambda^{2}\right)\left(\sigma_{q q}+\sigma_{p p}\right)+\frac{1}{4}\left(1-\lambda^{2}\right)^{2}\right) \geqslant \frac{1}{4 \lambda^{4}} \hbar^{2}, \\
\sigma_{q q \lambda} \sigma_{p p \lambda}-\sigma_{q p \lambda}^{2}=\frac{1}{\lambda^{4}}\left(\sigma_{q q} \sigma_{p p}-\sigma_{q p}^{2}+\frac{1}{2}\left(1-\lambda^{2}\right)\left(\sigma_{q q}+\sigma_{p p}\right)+\frac{1}{4}\left(1-\lambda^{2}\right)^{2}\right) \geqslant \frac{1}{4 \lambda^{4}} \hbar^{2} .
\end{array}
$$

Видно, что правые части данных соотношений неопределенностей содержат множитель $\lambda^{-4}$, поэтому при $|\lambda|<1$ их величина возрастает и, вообще говоря, может стать сколь угодно большой. Точно таким же свойством обладают и некоторые другие состояния, например так называемые коррелированные состояния, которые возникают как некоторые обобщения когерентных состояний [23]. Эти состояния обладают большой неопределенностью по координате и импульсу. В работах [24]-[26] было предложено использовать это их свойство для описания некоторых явлений, в которых наблюдается бо́льшая, чем обычно, вероятность прохождения частиц через потенциальный барьер. Увеличение вероятности туннельного перехода можно формально соотнести с "увеличением" постоянной Планка $\hbar$, т. е. считать, что преобразование масштаба $(q, p) \rightarrow(\lambda q, \lambda p)$ приводит к возникновению "эффективной постоянной Планка" $\hbar_{\mathrm{eff}}=\hbar / \lambda^{2}$. Для $\lambda^{2} \ll 1$ эффективная постоянная Планка удовлетворяет неравенству $\hbar_{\mathrm{eff}} \gg \hbar$.

\section{8. СЛУЧАЙ БОЛЬШИХ $\lambda$}

До сих пор при наших рассуждениях мы предполагали, что $\lambda<1$. При этом мы опирались на теорему, доказанную в работе [17]. Согласно этой теореме, если $Q(q, p)$ - функция Хусими квантового состояния и $\lambda<1$, то $\lambda^{2} Q(\lambda q, \lambda p)$ есть тоже функция Хусими некоторого квантового состояния. Для $\lambda>1$ никакие общие утверждения такого типа неизвестны. Чтобы понять, какие могут при этом возникнуть проблемы, попробуем реализовать для $\lambda>1$ ту же конструкцию, которая использовалась нами при $\lambda<1$.

Сначала рассмотрим случай $1<\lambda<2$. Выберем $N$-частичное фоковское состояние $|N\rangle$. Его функция Хусими такова:

$$
Q^{N}(q, p)=e^{-|\alpha|^{2}} \frac{|\alpha|^{2 N}}{N !}
$$

После преобразования $(q, p) \rightarrow(\lambda q, \lambda p)$ она принимает вид

$$
\lambda^{2} Q^{N}(\lambda q, \lambda p)=\lambda^{2+2 N} e^{-\lambda^{2}|\alpha|^{2}} \frac{|\alpha|^{2 N}}{N !}=e^{-4|\alpha|^{2}} \lambda^{2+2 N} e^{\left(4-\lambda^{2}\right)|\alpha|^{2}} \frac{|\alpha|^{2 N}}{N !} .
$$

Как и прежде, мы хотим представить данную функцию в виде суммы величин вида (78). С этой целью разложим экспоненту $e^{\left(4-\lambda^{2}\right)|\alpha|^{2}}$ в ряд:

$$
e^{\left(4-\lambda^{2}\right)|\alpha|^{2}}=\sum_{j=0}^{\infty} \frac{\left(4-\lambda^{2}\right)^{j}}{j !}|\alpha|^{2 j}
$$


Подставляя это разложение в формулу (79), получаем для $1<\lambda<2$

$$
\begin{aligned}
\lambda^{2} Q^{N}(\lambda q, \lambda p) & =\sum_{j=0}^{\infty} \frac{\left(4-\lambda^{2}\right)^{j}}{N ! j !} \lambda^{2+2 N} e^{-4|\alpha|^{2}}|\alpha|^{2 N+2 j}= \\
& =\sum_{j=0}^{\infty} \frac{\left(4-\lambda^{2}\right)^{j}}{N ! j !} \lambda^{2+2 N} e^{-4|\alpha|^{2}} \frac{\left(4|\alpha|^{2}\right)^{N+j}}{4^{N+j}}= \\
& =\sum_{j=0}^{\infty} \frac{\left(4-\lambda^{2}\right)^{j}(N+j) !}{N ! j !} \lambda^{2+2 N} \frac{1}{4^{N+j}}\left(e^{-4|\alpha|^{2}} \frac{\left(4|\alpha|^{2}\right)^{N+j}}{(N+j) !}\right)= \\
& =4 \sum_{j=0}^{\infty} \frac{(N+j) !}{N ! j !}\left(1-\frac{\lambda^{2}}{4}\right)^{j}\left(\frac{\lambda^{2}}{4}\right)^{1+N} Q^{N+j}(2 q, 2 p) .
\end{aligned}
$$

В общем случае, когда $s-1<\lambda<s$, имеем

$$
\lambda^{2} Q^{N}(\lambda q, \lambda p)=s^{2} \sum_{j=0}^{\infty} \frac{(N+j) !}{N ! j !}\left(1-\frac{\lambda^{2}}{s^{2}}\right)^{j}\left(\frac{\lambda^{2}}{s^{2}}\right)^{1+N} Q^{N+j}(s q, s p) .
$$

Как и прежде, коэффициенты при величинах $Q^{N+j}(s q, s p)$ в суммах $(81),(82)$ являются элементами отрицательного биномиального распределения. Их сумма равна единице, поэтому ряды (81), (82) сходятся, и остается открытым лишь вопрос об интерпретации величин

$$
Q^{N}(s q, s p)=s^{2 N} e^{-s^{2}|\alpha|^{2}} \frac{|\alpha|^{2 N}}{N !}=e^{-s^{2} q^{2}-s^{2} p^{2}} \frac{\left(s^{2} q^{2}+s^{2} p^{2}\right)^{N}}{N !} .
$$

В работе [27] изучался вопрос о том, каким условиям должна удовлетворять функция $F(q, p)$, заданная на фазовом пространстве, чтобы она являлась функцией Хусими квантового состояния. В этой работе было показано, что гауссова функция

$$
F(q, p)=C e^{-\lambda^{2} q^{2}-\lambda^{2} p^{2}}
$$

является функцией Хусими квантового состояния в том и только в том случае, когда $\lambda \leqslant 1$. Функции (83) не удовлетворяют этому критерию, и, следовательно, им не отвечает никакое квантовое состояние, т.е. они не являются функциями Хусими. Поэтому преобразование масштаба (3) при $\lambda^{2}>1$, вообще говоря, не переводит функцию Хусими в функцию Хусими.

\section{9. ЗАКЛЮЧЕНИЕ}

В представленной работе предложен новый подход к построению состояний, которые возникают в результате квантовых процессов. Этот метод основан на использовании квазивероятностных распределений, более конкретно, функций Хусими. Последовательность рассуждений такова. Пусть некоторый процесс описывается гамильтонианом $H$. Используя этот гамильтониан, можно найти операторное уравнение для матрицы плотности $\rho$. Используя связь между функцией Хусими $Q$ и матрицей плотности $\rho$, от этого операторного уравнения можно перейти к обыкновенному дифференциальному уравнению для функции Хусими. 
Суть нашего метода состоит в том, что решениям данного дифференциального уравнения сопоставляется преобразование фазового пространства. Это преобразование индуцирует преобразование заданных на нем функций Хусими. Выражая такие преобразованные функции $\widetilde{Q}$ через уже известные функции Хусими, можно осуществить обратный переход и найти матрицу плотности $\tilde{\rho}$ преобразованного состояния. В нашей работе эта программа была реализована для преобразования масштаба (3) в фазовом пространстве, которое моделирует действие квантового усилителя [18], [19]. Была найдена матрица плотности растянутого состояния, возникающего в результате воздействия такого усилителя на $n$-частичные состояния гармонического осциллятора и их произвольные суперпозиции. В дальнейшем мы предполагаем рассмотреть другие состояния и другие преобразования фазового пространства, в частности такие, которые приводят не к усилению, а к ослаблению фоковских состояний.

Благодарности. В. А. Андреев благодарит за гостеприимство Vinča Institute of Nuclear Sciences (Белград, Сербия), а Д. М. Давидович благодарит за гостеприимство Физический институт им. П. Н. Лебедева РАН.

\section{Список литературы}

[1] E. P. Wigner, "On the quantum correction for thermodynamic equilibrium", Phys. Rev., 40:5 (1932), 749-759.

[2] K. Husimi, "Some formal properties of the density matrix", Proc. Phys. Math. Soc. Japan. Ser. 3, 22:4 (1940), 264-314.

[3] Y. Kano, "A new phase-space distribution function in the statistical theory of the electromagnetic field", J. Math. Phys., 6:12 (1965), 1913-1916.

[4] R. J. Glauber, "Photon correlations", Phys. Rev. Lett., 10:3 (1963), 84-86.

[5] E. C. G. Sudarshan, "Equivalence of semiclassical and quantum mechanical descriptions of statistical light beams", Phys. Rev. Lett., 10:7 (1963), 277-278.

[6] Р. Л. Стратонович, "О распределениях в изображающем пространстве”, ЖЭТФ, 31:6 (1956), 1012-1020; "Калибровочно-инвариантный аналог распределения Вигнера", Докл. АН СССР, 109:1 (1956), 72-75.

[7] K.E. Cahill, R. J. Glauber, "Ordered expansions in boson amplitude operators", Phys. Rev., 177:5 (1968), 1857-1881; "Density operators and quasiprobability distributions", Phys. Rev., 177:5 (1969), 1882-1902.

[8] M. Hillery, R. F. O'Connell, M. O. Scully, E. P. Wigner, "Distribution functions in physics: fundamentals", Phys. Rep., 106:3 (1984), 121-167.

[9] В.И. Татарский, "Вигнеровское представление квантовой механики", УФН, 139:4 (1983), 587-619.

[10] А. С. Холево, Вероятностные и статистические аспекты квантовой теории, ИКИ, М., Ижевск, 2003.

[11] L. Mandel, E. Wolf, Optical Coherence and Quantum Optics, Cambridge Univ. Press, Cambridge, 1995.

[12] M. O. Scully, M. S. Zubairy, Quantum Optics, Cambridge Univ. Press, Cambridge, 1997.

[13] W. P. Schleich, Quantum Optics in Phase Space, Wiley, Berlin, 2001.

[14] A. B. Klimov, S. M. Chumakov, A Group-Theoretical Approach to Quantum Optics, Wiley, Weinheim, 2009.

[15] S. Mancini, V.I. Man'ko, P. Tombesi, "Wigner function and probability distribution for shifted and squeezed quadratures", Quantum Semiclass. Opt., 7:4 (1995), 615-623; "Symplectic tomography as classical approach to quantum systems", Phys. Lett. A, 213:1-2 (1996), 1-6; "Classical-like description of quantum dynamics by means of symplectic tomography", Found. Phys., 27:6 (1997), 801-824. 
[16] A. Ibort, V. I. Man'ko, G. Marmo, A. Simoni, F. Ventriglia, "An introduction to the tomographic picture of quantum mechanics", Phys. Scr., 79:6 (2009), 065013, 29 pp.

[17] В. А. Андреев, Д. М. Давидович, Л. Д. Давидович, М. Д. Давидович, В. И. Манько, М. А. Манько, "Трансформационное свойство функции Хусими и ее связь с функцией Вигнера и симплектическими томограммами", ТMФ, 166:3 (2011), 410-424.

[18] G. S. Agarwal, K. Tara, "Transformations of the nonclassical states by an optical amplifier", Phys. Rev. A, 47:4 (1993), 3160-3166.

[19] G. S. Agarwal, S. Chaturvedi, A. Rai, "Amplification of maximally-path-entangled number states", Phys. Rev. A, 81:4 (2010), 043843, 5 pp.

[20] V.A. Andreev, P. B. Lerner, "Supersymmetry in the Jaynes-Cummings model", Phys. Lett. A, 134:8-9 (1989), 507-511.

[21] В. Феллер, Введение в теорию вероятностей и ее приложения, т. 1, Мир, М., 1984.

[22] В.А. Андреев, Л.Д. Давидович, Милена Д. Давидович, Милош Д. Давидович, В. И. Манько, М. А. Манько, "Операторный метод вычисления $Q$-символов и их связь с символами Вейля-Вигнера и символами симплектических томограмм", ТМФ, 179:2 (2014), 207-224.

[23] В. В. Додонов, В.И. Манько, Инварианты и эволюиия нестационарных квантовых систем, Тр. ФИАН СССР, 183, Наука, М., 1987.

[24] В.И.Высоцкий, М. В. Высоцкий, С. В. Адаменко, “Особенности формирования и применения коррелированных состояний в нестационарных системах при низкой энергии взаимодействующих частиц", ЖЭТФ, 141:2 (2012), 276-287.

[25] В. И. Высоцкий, М. В. Высоцкий, С. В. Адаменко, “Формирование коррелированных состояний и увеличение прозрачности барьера при низкой энергии частиц в нестационарных системах с демпфированием и флуктуациями", ЖЭТФ, 142:4 (2012), 627-643.

[26] V. N. Chernega, Purity dependent uncertainty relation and possible enhancement of quantum tunneling phenomenon, arXiv: 1303.5238.

[27] D. M. Davidović, D. Lalović, "When does a given function in phase space belong to the class of Husimi distributions?", J. Phys. A: Math. Gen., 26:19 (1993), 5099-5106.

Поступила в редакцию 18.05.2016, после доработки 11.07.2016 